\title{
Low Complex, Programmable FPGA based 8-Channel Ultrasound Transmitter for Medical Imaging Researches
}

\author{
Chandrashekar Dusa ${ }^{1}$, P. Rajalakshmi ${ }^{1}$, Suresh Puli ${ }^{1}$, U. B. Desai ${ }^{1}$, S. N. Merchant ${ }^{2}$ \\ ${ }^{1}$ Department of Electrical Engineering, Indian Institute of Technology Hyderabad, India \\ Email: $\{$ ee12m1014, raji, sureshpuli\}@iith.ac.in \\ ${ }^{2}$ Department of Electrical Engineering, Indian Institute of Technology Bombay, India \\ Email: merchant@ee.iitb.ac.in
}

\begin{abstract}
In commercial ultrasound systems, the transmit module typically generates the time delayed excitation pulses to steer and focus the acoustic beam. However, the ultrasound transmitter module in these systems has limited access to medical ultrasound researchers. In this paper, we have presented the development of a programmable architecture for 8-channel ultrasound transmitter for medical ultrasound research activities. The proposed architecture consists of 8 transmit channels and Field Programmable Gate Array (FPGA) based configurable delay profile to steer acoustic beam, transmit frequency and pulse pattern length depending on the medical application. Our system operates in pulse-echo mode, with ultrasound transmit frequency up to $20 \mathrm{MHz}$, excitation voltage up to $100 \mathrm{Vpp}$, and individual channel control with single high speed Serial Peripheral Interface (SPI). Pre-calculated delay profiles per scanline are generated in Matlab, based on physical parameters of 8 element linear transducer array which are used to steer and focus the ultrasound beam. An experiment is carried with our transmit module to transmit ultrasound into gelatin phantom, acquired echoes and processed for B-mode imaging. The results show that this transmit platform can be used for ultrasound imaging researches and also for medical diagnosis.
\end{abstract}

Index Terms - Ultrasonic imaging, ultrasonic transducer array, field programmable gate array, transmit beamformer, high voltage pulser

\section{INTRODUCTION}

Ultrasound is radiation-free, patient-friendly and lessexpensive when compared to other medical imaging techniques. The adoption of this modality by all categories of hospitals and other health care institutions has given rise to new designs and market opportunities [1]. In modern ultrasound imaging systems, the ultrasound transmit module consists of digital Transmit (Tx) beamformer typically generates necessary logic pulses with proper timing and phase to enable electronic steer and focus on the acoustic beam. However, these systems often "closed" architecture provides the researchers to have limited access to the ultrasound transmit module [2].

Recently, Amauri et al. in [3] discussed the development of programmable FPGA based 8 independent channel Arbitrary Waveform Generator (AWG) for medical ultrasound research activities. However, this AWG transmit platform requires additional expensive electronics includes high voltage MOSFET drivers, Transformers. The digital Tx beamformer is configured using FPGA device for accurate control on transmission parameters such as center frequency and pulse pattern length to optimize image quality based on the medical application. FPGAs improve the ability for ultrasound imaging systems to create small form factor and high-performance products with reduced power consumption [4]. In [5], Gabriella et al. have proposed a new beamforming technique in which the transmit aperture apodization by varying the length of the excitation pulses.

The spatial resolution of a B-mode image can be evaluated into lateral resolution and axial resolution. It represents the smallest distance, the reflectors can be separated and still be distinguishable as separate points [6]. Higher frequencies are in principle more desirable, since they provide higher resolution but limited by tissue attenuation [7]. Short ultrasound pulses are required for better lateral resolution of image [8]

This paper presents the design of a programmable FPGA based 8-channel "ultrasound transmit module" for medical ultrasound researches. Our design uses Spartan 3E FPGA to configure the digital $\mathrm{Tx}$ beamformer with single high speed 4-wire serial interface for transmission parameters. Depending on the medical applications user can configure Tx parameters such as delay profile for acoustic beam steering, transmit frequency, and pulse length. Pre-calculated delay profile is updated to Tx beamformer per each transmission in different steering angles. We have conducted an experiment by transmitting ultrasound into gelatin phantom. The electrical signals of echoes from each focal point are acquired by AFE module and further applied to signal processing algorithms for ultrasound imaging.

This paper is organized as follows, section II introduces the ultrasound transmit system architecture, section III discusses the hardware setup for proposed architecture and observed results. Conclusions and future work are discussed in section IV.

\section{SYSTEM DESCRIPTION}

Fig. 1 shows the block diagram for ultrasound imaging system architecture. The architecture mainly consists of transducer array, High Voltage (HV) pulser, digital Tx beamformer, FPGA device, user interface, Analog Front End (AFE) and signal processing modules. The basic principle for an ultrasound imaging system is to transmit ultrasound burst signal into the area of interest of organ, receive echoes and 
process for imaging [9]. The proposed design of ultrasound transmitter reflects the same principle with user interface to enable flexibility in modifying the transmission parameters. Here digital Tx beamformer is configured using SPI controlling signals from FPGA. With FPGA device, we programmed the internal registers of $\mathrm{Tx}$ beamformer to change delay profile for each channel, frequency of diagnosis and pulse length depending on the medical application.

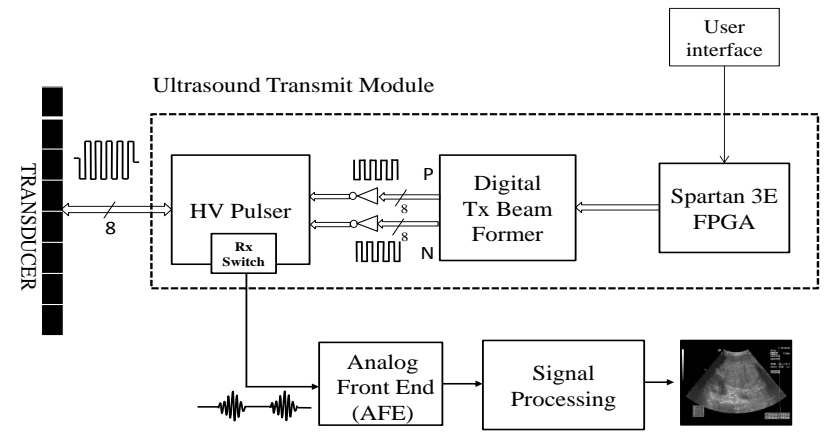

Fig. 1: Block diagram of ultrasound imaging system architecture

\section{A. Transducer array}

The medical ultrasound imaging transducers are excited in two modes: linear and phased array mode. In linear array mode, subset of transducer elements are excited where as in phased array mode all the elements of transducer are excited to focus the sharp ultrasound beam at focal point. For a transducer array, the piezoelectric element pitch size is required to be smaller than half of the wavelength [10]. To scan interest of organ, the ultrasound beam should be focused at multiple focal points. The time delays are applied to excitation of sensor elements to create constructive interference of wave fronts at different focal points. The basic principle of phased array transducer excitation is shown in Fig. 2.

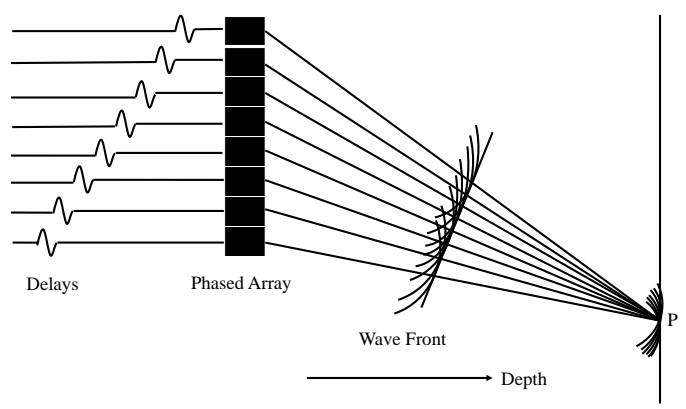

Fig. 2: Principle of phased array transducer

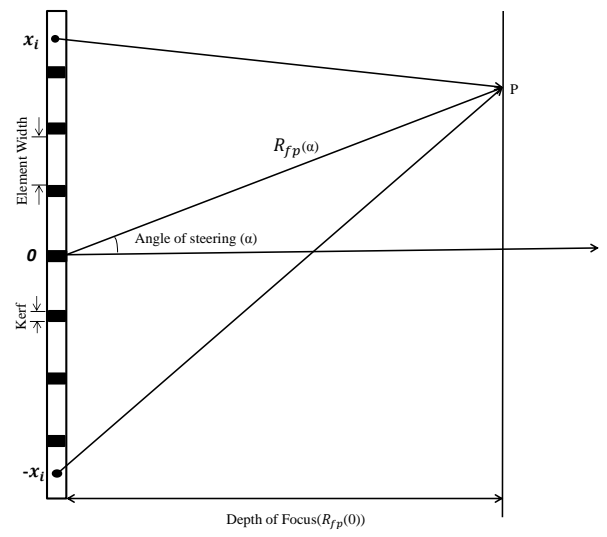

Fig. 3: Calculations of delay profile for a steering angle $\left(\alpha^{\circ}\right)$

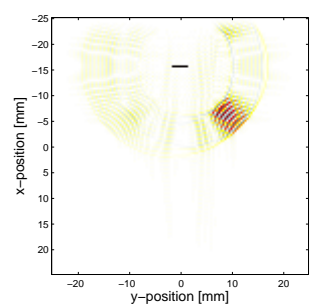

(a) $+30^{\circ}$ steering of pressure wave distribution

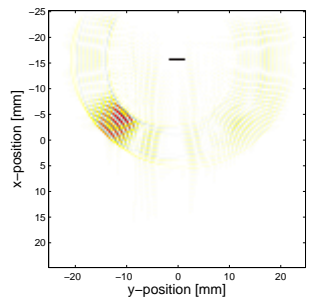

(c) $-30^{\circ}$ steering of pressure wave distribution

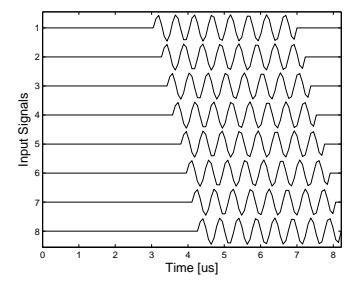

(b) Phased excitation signals for 8transducer elements

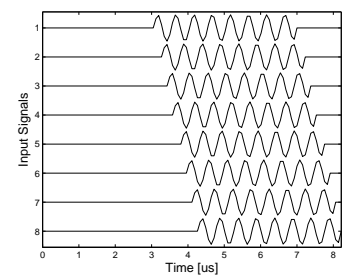

(d) Phased excitation signals for 8transducer elements
Fig. 4: Simulation results

The time delays can be obtained using simple geometry as illustrated in Fig. 3 [11]. Average ultrasound velocity in tissue is considered as $1540 \mathrm{~m} / \mathrm{s}$. First, each element is treated as a point source located at the geometric center of the physical element. As the delays used in transmit beamforming are additive delays, the difference between these distances and the distance from the outermost element is then calculated. This gives the difference in travel distance for the acoustic waves that must be corrected for using electronic delays.

$$
t_{i}=\frac{\sqrt{R_{f p(\alpha)}^{2}+x_{i}^{2}-2 x_{i} R_{f p(\alpha)} \sin (\alpha)}}{c}
$$

where $R_{f p(\alpha)}=\frac{R_{f p(0)}}{\cos (\alpha)} \frac{a}{b}$

$$
T_{i}=t_{\max }-t_{i}
$$




$$
\begin{aligned}
P \rightarrow & \text { Focal point. } \\
R_{f p(\alpha)}, R_{f p(0)} \rightarrow & \text { Distance from center element to point } \mathrm{P} \\
t_{i} \rightarrow & \text { Time required for wave front to } \\
& \text { reach point } \mathrm{P} . \\
x_{i} \rightarrow & \text { Co-ordinate of } i^{t h} \text { element. } \\
t_{\max } \rightarrow & \text { Max time required for wave front to } \\
& \text { reach point } \mathrm{P} . \\
T_{i} \rightarrow & \text { Delay for } i^{t h} \text { element. }
\end{aligned}
$$

The proper time delays for beam steering are obtained from equation (1) and (2). These delay profile per scanline at a given steering angle are simulated using software tool. Fig. 4 shows pressure wave distribution in 2-D and phase delays of burst signal for each transducer element for $+30^{\circ}$, $-30^{\circ}$ respectively.

\section{B. FPGA Based Acoustic Beam Steering}

Our design uses Spartan-3E FPGA starter kit [12] to control the direction of acoustic beam and depth of focus. To steer and focus the beam, each transducer element is delayed and excited by $T_{i}$ calculated from equation (1) and (2). A single high speed Serial Peripheral Interface (SPI) between the FPGA and the Tx beamformer, achieves fast update of delay profile per each scanline. It can configure frequency of operation and pulse length to optimize the image quality. The FPGA will also generate pulse repetition rate control signal of frequency based on depth of interest [13]. Pre-calculated delay profile per scanline are stored in FPGA register block memory to reduce the computational burden [14].

\section{Digital Tx Beamformer}

The proposed design uses a 8-channel programmable beamformer to generate proper phased logic pulses by programming with FPGA. The beamformer has internal programmable registers, which can be programmed to configure transmission parameters at a maximum data rate of $80 \mathrm{Mbps}$ [15]. The Tx beamforming technique is to generate time delayed logic inputs to focus ultrasound beam at focal point, this improves resolution of image. The level of improvement in resolution of ultrasound image depends on minimum time delay of Tx beamformer. The phased logic pulses of two different channels with 64 pulse pattern length are shown in Fig. 5.

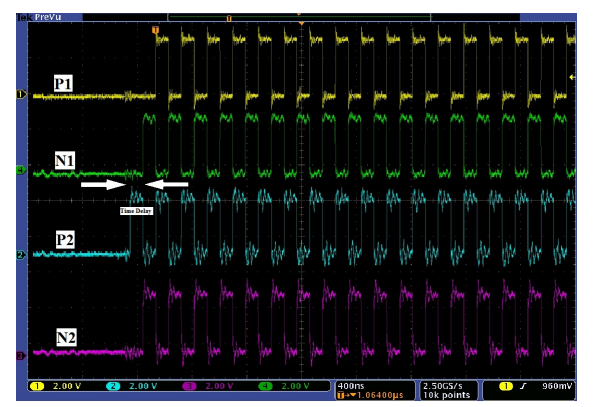

Fig. 5: Logic pulses of two channels

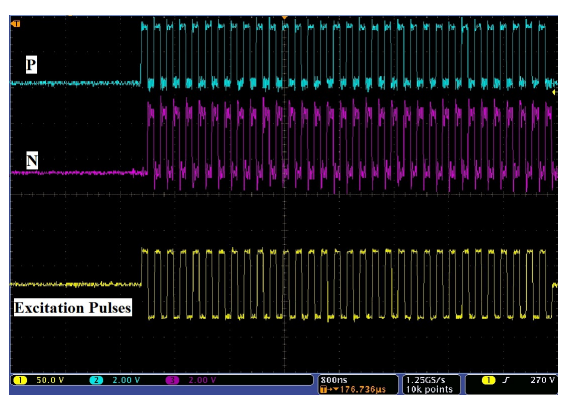

Fig. 6: $\mathrm{HV}$ pulses with $\mathrm{P}$ and $\mathrm{N}$ logics

\section{HV pulser}

The HV pulser simply acts as Digital to Analog Converter (DAC) to amplify the digital outputs of beamformer. Typically the ultrasound sensors are excited at high voltage (100 Vpp) and high frequencies (2-20 MHz) [16]. The HV pulser used in the proposed architecture can also acts as switch to receive ultrasound reflected signal from tissue. Two logic inputs of pulser controls the chip functionality as Rx switch and DAC amplifier. If two inputs are logic one then the pulser circuit can act as receive switch to receive low voltage signals of reflected ultrasound waves and prevent overloading of AFE module. If two inputs are logic zero then output of pulser is zero. When two inputs are different logic then it generates bipolar high voltages. Fig. 6 shows $\mathrm{P}$ and $\mathrm{N}$ logic pulses from the beamformer and corresponding high voltage signal.

\section{E. User interface}

A Graphical User Interface (GUI) shown in Fig. 7 has been developed with National Instruments LabVIEW 2012 in the Microsoft Windows platform. Spartan-3E starter kit is connected to Personal Computer (PC) through USB and interfaced LabVIEW project. This can provide extensive user control of transmission parameters during pulse-echo experiments. Multiple transmission parameters for each of 8 channels can be modified, including coarse time delay and fine time delay. Other parameters, such as operating frequency of ultrasound, pulse pattern length, pulse repetition rate are selected for all channels. All these parameters can be saved and loaded for B-mode imaging researches.

\section{F. Main features of the proposed ultrasound transmit plat-} form

- Programmable 8 independent transmit channels to drive eight transducer elements, which can be extended to more number of channels.

- High speed serial interface for 8-channel with data rate up to $80 \mathrm{Mbps}$.

- Flexible user interface to select and modify transmission parameters.

- Maximum ultrasound transmit output bandwidth 80 MHz.

- Supports 4-64 bit programmable pulse pattern.

- Minimum time delay of beamformer $0.78 \mathrm{~ns}$. 


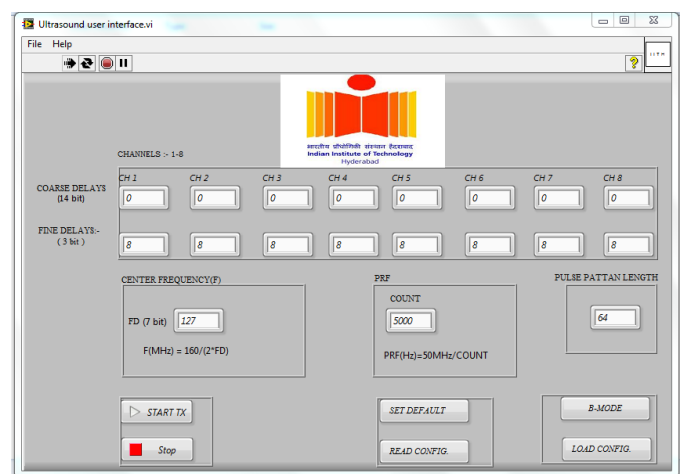

Fig. 7: User interface to control the transmission parameters

- The HV pulser also features 8-integrated transmit receive (T/R) switches.

- Flexibility to examine the output signals at each stage of the system.

- Our design for 8-channel transmit module is a low complex hardware and inexpensive $(\simeq \$ 166)$ compared to existing transmit modules for research purposes.

\section{G. Comparative study with literature}

Our design exploits the latest digital electronic technology to realize a low hardware complex, relatively inexpensive ultrasound transmit system. The existing transmit platforms in the literature are using additional expensive electronics for each channel [3]. The hardware of our proposed Tx architecture is optimized with the usage of high performance 8-channel Application Specific Integrated Circuits (ASICs) for Tx beamformer and HV pulser as shown in Fig. 1. A flexible user interface as shown in Fig. 7 is provided with accurate control of transmission parameters for ultrasound transmit researches.

\section{EXPERIMENTAL RESULTS}

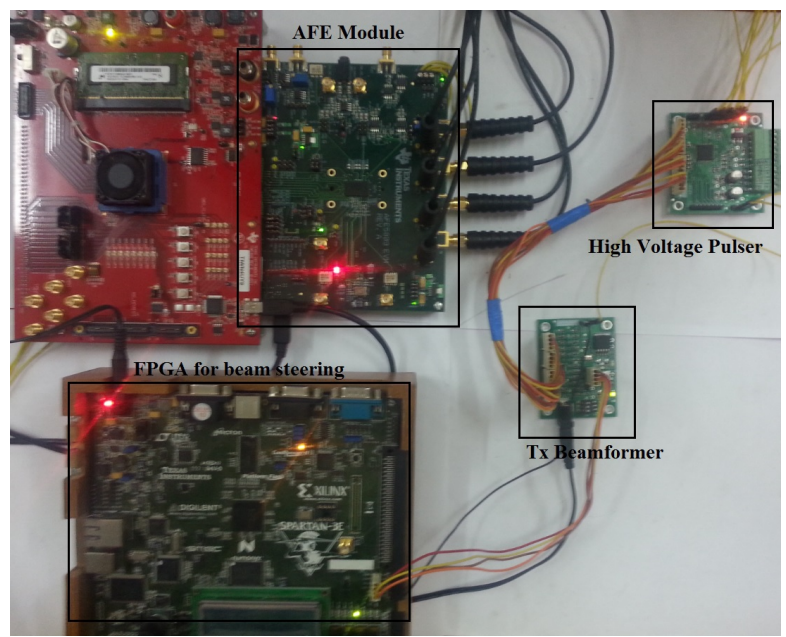

Fig. 8: Programmable 8-channel ultrasound transmit module

The complete prototype of programmable ultrasound transmit module is shown in Fig. 8. The overall hardware consists of two in-house made boards for Tx beamformer and HV pulser, Spartan 3E starter board, and AFE 5809 EVM. The spartan 3E starter board is connected to any PC through USB 2.0, where a custom software runs as user interface.

An experiment is conducted using our design to transmit ultrasound burst in to the gelatin phantom to acquire B-mode image. The transducer parameters and experiment settings are mentioned in table I. Pre-calculated delay profile to steer and focus the acoustic beam are loaded into GUI. The delay profile to 8-channels are dynamically updated to $\mathrm{Tx}$ beamformer between each consecutive transmission in different steering angles.

TABLE I: Experiment and transducer specifications

\begin{tabular}{|c|c|}
\hline Specification & value \\
\hline Transmit frequency & $5 \mathrm{MHz}$ \\
\hline Excitation voltage & $100 \mathrm{Vpp}$ \\
\hline Number of elements & 8 \\
\hline Kerf of transducer & $.025 \mathrm{~mm}$ \\
\hline Element width & $.154 \mathrm{~mm}$ \\
\hline Imaging depth & $50 \mathrm{~mm}$ \\
\hline Field of view & $-60^{\circ}$ to $+60^{\circ}$ \\
\hline
\end{tabular}

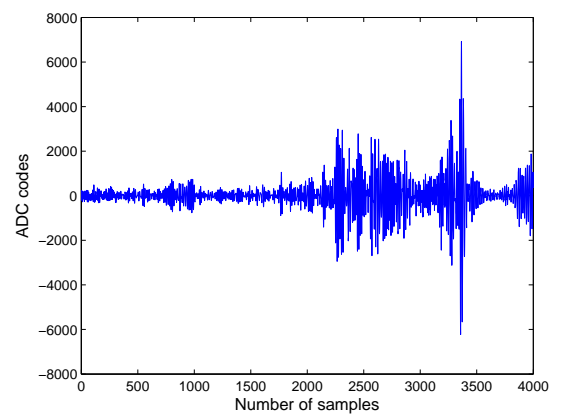

Fig. 9: RF Scanline data

The reflected signals from the transducer at output of pulser as shown in Fig. 1 are further processed to filter noise and digitization by AFE module. In our design AFE 5809 Evaluation Module (EVM) [17] is used to acquire low voltage signals, time gain compensation, filtering, and digitizing, where as output samples are in Low Voltage Differential Signal (LVDS) format. The LVDS output data of AFE is de-serialized using FPGA and taken to a commercial PC to apply signal processing algorithms for ultrasound image.

The acquired Radio Frequency (RF) data samples from programmable transmit module as shown are further processed for receive beamforming, demodulation, signal processing to display ultrasound image. We have implemented receive beamforming Delay And Sum (DAS) algorithm [18]. In this algorithm the received echoes from 8 transducer elements for given depth, compensate their phase for different paths, and then summed to form receive beam. Fig. 9 shows single scanline data samples after applying receive beamforming algorithm to sensor data. The signal processing 


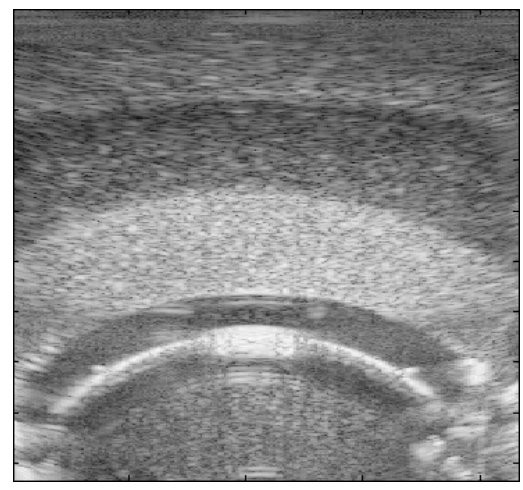

Fig. 10: Reconstructed ultrasound image of gelatin phantom

includes envelop detection, log compression, interpolation and scan conversion. Fig. 10 shows the reconstructed Bmode image of gelatin phantom acquired using proposed low complex prototype of ultrasound transmit module.

\section{CONCLUSION}

In this paper, we have presented the development of a programmable 8-channel ultrasound transmit module for medical ultrasound research activities. Researchers can use our design to implement new receive beamforming and signal processing algorithms to optimize the image quality with extensive user control of transmission parameters. A user interface was developed to control time delays, pulse repetition frequency (PRF), pulse pattern length and operating frequency. The FPGA based ultrasound transmit platform was developed using a reasonably inexpensive FPGA starter board, two in-house made boards for Tx beamformer and HV pulser. The proposed ultrasound transmit module prototype was tested by transmitting ultrasound into gelatin phantom for B-mode imaging. Reconstructed image from the data acquired through the prototype was good enough for ultrasound researches and medical diagnosis.

The further optimization of transmit beamforming technique and user interface is necessary to facilitate the development and test of more transmit techniques such as Continuous Wave Doppler (CWD).

\section{ACKNOWLEDGEMENT}

This project is funded by Department of Science and Technology (DST) under IU-ATC IoT e-health project. We would like to thank Mr. Pradeep Mishra for helping in user interface for the system.

\section{REFERENCES}

[1] Chiang, A.M.; Chang, P.P.; Broadstone, S.R., "PC-based ultrasound imaging system in a probe," Ultrasonics Symposium, 2000 IEEE , vol.2, no., pp. 1255,1260 vol.2, Oct 2000.

[2] Bassi, L.; Boni, E.; Cellai, A.; Dallai, A.; Guidi, F.; Ricci, S.; Tortoli, P., "A Novel Digital Ultrasound System for Experimental Research Activities," Digital System Design Architectures, Methods and Tools, 2008. DSD '08. 11th EUROMICRO Conference on, vol., no., pp.413,417, 3-5 Sept. 2008.
[3] Assef, A.A.; Maia, J.M.; Schneider, F.K.; Costa, E.T.; da Silveira Nantes Button, V.L., "A programmable FPGA-based 8-channel arbitrary waveform generator for medical ultrasound research activities," Engineering in Medicine and Biology Society (EMBC), 2012 Annual International Conference of the IEEE, vol., no., pp.515,518, Aug. 28 2012-Sept. 12012.

[4] Jon Alexander, "Xilinx Devices in Portable Ultrasound Systems", WP378 (v1.2) May 13, 2013.

[5] Cincotti, G.; Cardone, G.; Gori, P.; Pappalardo, M., "Efficient transmit beamforming in pulse-echo ultrasonic imaging," Ultrasonics, Ferroelectrics and Frequency Control, IEEE Transactions on, vol.46, no.6, pp.1450,1458, Nov. 1999.

[6] Kouame, D.; Ploquin, M., "Super-resolution in medical imaging : An illustrative approach through ultrasound," Biomedical Imaging: From Nano to Macro, 2009. ISBI '09. IEEE International Symposium on , vol., no., pp.249,252, June 28 2009-July 12009.

[7] Eberhard Brunner, "How Ultrasound System Considerations Influence Front-End Component Choice". Analog Dialogue 36-03 (2002).

[8] Alexander Ng MB ChB FRCA MD, Justiaan Swanevelder MB ChB FRCA FCA(SA) MMed, "Resolution in ultrasound imaging", Oxford University Press on behalf of the British Journal of Anaesthesia, August 2011.

[9] Vincent Chan and Anahi Perlas, "Basics of Ultrasound Imaging", S.N. Narouze (ed.), Atlas of Ultrasound-Guided Procedures in Interventional Pain Management, DOI 10.1007/978-1-4419-1681-5_2, Springer Science+Business Media, LLC 2011.

[10] R. K. Saha, S. Karmakar, S. Saha, M. Roy, S. Sarkar and S.K. Sen Microelectronics Division, Saha Institute of Nuclear Physics, 1/AF Bidhannagar, Kolkata-700064, "Ultrasonic Linear Array Medical Imaging System".

[11] Texas Instruments, "Signal Processing Overview of Ultrasound Systems for Medical Imaging ", SPRAB12 November 2008.

[12] Xilinx, "Spartan-3E Starter Kit Board User Guide", UG230 (v1.0) March 9, 2006.

[13] Chris Basoglu, Ravi Managuli, George York, Yongmin Kim, "Computing requirements of modern medical diagnostic ultrasound machines" Parallel Computing Vol. 24, Sep. 1998.

[14] Schneider, F.K.; Anup Agarwal; Yang Mo Yoo; Fukuoka, T.; Yongmin Kim, "A Fully Programmable Computing Architecture for Medical Ultrasound Machines," Information Technology in Biomedicine, IEEE Transactions on, vol.14, no.2, pp.538,540, March 2010.

[15] Texas Instruments, "LM96570 Ultrasound Configurable Transmit Beamformer", SNAS505D, September 15, 2011.

[16] Maxim Integrated Products, Inc., "MAX14808/MAX14809 Octal Three-Level/Quad Five-Level High-Voltage 2A Digital Pulsers with T/R Switch", 19-6438, Rev 1, March 2013.

[17] Texas Instruments, AFE5809EVM, available online at http: //www.ti.com/ww/en/analog/AFE5809_ultrasonic_ AFE_with_digital_demodulator/index. shtml ?DCMP $=$ corp_gmd_afe5809_en\&HQS=gmd-afe5809-bthi-en.

[18] Sverre Holm, Vingmed Sound AS, Research Department, Vollsveien 13C, N-1324 Lysaker, Norway, Department of Informatics, University of Oslo, Norway, "Digital Beamforming In Ultrasound Imaging". 